\title{
Germen de tarificación de las sanciones ambientales
}

The environmental sanction's pricing seed

Claudio Tapia Alvial*

En este trabajo se analiza la decisión de la Corte Suprema en el caso FMC Ltda. con la Superintendencia del Medio Ambiente. Vinculamos la decisión con las líneas jurisprudenciales atingentes y argumentamos que implica algunos efectos perjudiciales e.g., la tarificación de las sanciones ambientales.

Palabras clave: Derecho Ambiental, Sanción Ambiental, Determinación de las sanciones ambientales.
This paper examines the Supreme Court decision in the case of FMC Ltd. v. Superintendency of the Environment. We link the decision with the pertinent jurisprudential rulings and argue that imply some adverse effects e.g., the environmental sanction's pricing.

Keywords: Environmental Law, Environmental Sanction, Determination of environmental sanctions.

\section{Introducción}

El 26 de abril del 2021 la Corte Suprema dictó sentencia en el caso Fuenzalida Moure Compañía Limitada o FMC Ltda. con Superintendencia del Medio Ambiente ("caso FMC"), rechazando los recursos de casación en el fondo interpuestos por ambas partes y dejó firme la decisión del Segundo Tribunal Ambiental, la cual acogió parcialmente la reclamación de FMC Ltda ("FMC") y anuló parcialmente la sanción impuesta por la Superintendencia del Medio Ambiente ("SMA").

La antedicha decisión de la Corte Suprema es relevante pues, a juicio de este comentarista, si bien implica una continuación respecto de una serie de precedentes de la Corte Suprema que elevan el estándar de la motivación de los actos administrativos, también representa un quiebre a la jurisprudencia de los tribunales ambientales en materia de la determinación de las sanciones por parte de la SMA, pues exige

\footnotetext{
* Licenciado en Ciencias Jurídicas y Sociales de la Universidad de Chile y MSc Philosophy and Public Policy de la London School of Economics. Correo electrónico: c.s.tapia@lse. ac.uk/tapclaudio@gmail.com. Dirección postal: Miguel Claro 070, departamento 201 b, Providencia, Región Metropolitana, Chile.
}

Artículo recibido el 19 de agosto de 2021 y aceptado el 8 de noviembre de 2021. 
que se expliciten los puntajes o valores numéricos asignados a cada circunstancia considerada en la sanción. Estándar que, en caso de adoptarse de manera generalizada, puede implicar ciertos efectos perjudiciales.

Este artículo seguirá la siguiente estructura. Primero, se sintetizarán los principales elementos del caso, enfocándonos en aquellos vinculados a la determinación de la sanción y siguiendo el orden cronológico de la controversia (I), vale decir, la decisión de sancionar de la SMA (I.1), la sentencia del Segundo Tribunal Ambiental (I.2) y de la Corte Suprema (I.3). Segundo, se explicarán algunos elementos regulatorios respecto de la regulación de la determinación de las sanciones ambientales (II). Tercero, se vinculará la decisión del caso con otros precedentes judiciales relevantes (III). Cuarto, se identificará el principal riesgo que se avizora a partir de la decisión, esto es, un principio o germen de tarificación ambiental y sus consecuencias (IV). Finalmente, se sintetizarán las principales conclusiones a las que se ha arribado (V).

\section{Síntesis del caso}

\section{Decisión de la SMA}

La SMA mediante su Resolución Exenta N² 241 del 26 de febrero de 2018 ("Res. Ex. N²41/2018"), decidió sancionar a FMC por dos infracciones clasificadas como grave y leve. Una vez consideradas las circunstancias del artículo 40 de la Ley Orgánica de la SMA ("LOSMA"), se determinó una infracción de multa para la primera infracción de 88 unidades tributarias anuales ("UTA") y para la segunda de 20 UTA.

El tipo de análisis que realizó la SMA a propósito de la ponderación de las circunstancias del artículo 40 de la LOSMA fue el estándar para tal clase de resoluciones, esto es, se estructuró conforme al polinomio para la determinación de sanciones establecido en las Bases Metodológicas para la determinación de sanciones ambientales ("Bases Metodológicas").

De acuerdo con las Bases Metodológicas la sanción corresponde a la sumatoria del beneficio económico y el componente de afectación. Este último, se determina por una serie de multiplicadores que se componen por el valor de seriedad, los factores de disminución e incremento y el tamaño económico.

Figura 1: Polinomio de cálculo de las sanciones ambientales

\begin{tabular}{|c|}
\hline Sanción $=$ Beneficio Económico + Componente de Afectación $(C A)$ \\
\hline $\mathrm{CA}=\left(\right.$ Valor de seriedad $\times\left(1+\sum(\right.$ Factores de incremento $)-\sum($ Factores de disminución $\left.\left.)\right)\right) \times$ \\
Factor Tamaño económico
\end{tabular}

Fuente: Figura 3.1. de las Bases Metodológicas de la SMA.

En tal sentido, la sanción primero abordó el beneficio económico ${ }^{1}$ el cual se cuantificó numéricamente, luego el componente de afectación y a

\footnotetext{
1 Art. 40 c) LOSMA.
} 
propósito de aquél, el valor de seriedad, los factores de incremento y disminución, y la capacidad económica del infractor. Esta última se categorizó previa consideración numérica de los ingresos por venta anuales de la empresa.

Por su parte, el valor de seriedad se descompuso en un análisis de la importancia del daño causado o peligro ocasionado ${ }^{2}$, número de personas cuya salud pudo afectarse ${ }^{3}$ y la importancia de la vulneración al sistema jurídico de protección ambiental ${ }^{4}$.

El desarrollo de las circunstancias del artículo 40 de la LOSMA agrupadas a propósito del valor de seriedad, como de los factores de incremento y disminución, siguió la siguiente estructura de análisis: identificar el contenido de la circunstancia de manera alineada con su tratamiento sustantivo en las Bases Metodológicas, luego concretizar el análisis con los antecedentes propios del caso, finalizando con una conclusión que establece si se aplica o no la circunstancia analizada, todo ello sin identificar un puntaje o valoración numérica para las circunstancias que se estimaron procedentes. Sin embargo, en el caso de la vulneración al sistema jurídico de protección ambiental se expresó también la entidad de la vulneración, que en el caso fue descrita como media.

FMC dedujo recurso de reposición en contra de la sanción, la cual fue rechazada por la autoridad íntegramente.

\section{Sentencia del Segundo Tribunal Ambiental}

En contra de la sanción de la SMA, FMC dedujo recurso de reclamación, indicando en lo atingente a este comentario, que no se fundamentó debidamente el modo de determinar la sanción específica. Ello pues, solo respecto del beneficio económico se estableció el modo específico en que la circunstancia influyó en el monto de la multa, en las demás la resolución se limitó a indicar las circunstancias que serían descartadas o consideradas, pero sin precisar su influencia en el monto de la multa.

El Segundo Tribunal Ambiental acogió parcialmente la reclamación deducida por FMC en contra de la resolución sancionatoria. En concreto, anuló lo dispuesto en el capítulo VIII de la sanción relativo a la determinación de la sanción impuesta.

A propósito de la fundamentación de las circunstancias del artículo 40 de la LOSMA, el Segundo Tribunal Ambiental reprochó que la sanción al razonar sobre el componente de afectación no señaló el valor o puntaje que se asignó a los factores del valor de seriedad, ni a los factores de incremento o disminución,

\footnotetext{
2 Art.40 a) LOSMA.

${ }^{3}$ Art.40 b) LOSMA.

${ }^{4}$ Art.40 i) LOSMA.
} 
el acto reclamado se limita a señalar las razones para considerar tales factores y concluye si serán o no aplicados para determinar la sanción, sin dar cuenta de la medida en que estos influyen en el cálculo del componente de afectación ${ }^{5}$.

A su vez, haciendo eco de planteamientos doctrinales enfatizó que la "dictación de las bases metodológicas implica la existencia de un mayor estándar de fundamentación y, en consecuencia, una revisión judicial más intensa"6.

Además, señaló que:

la SMA debe fundamentar la ponderación de las circunstancias del artículo 40 de la LOSMA que le permiten arribar a la sanción específica aplicada al caso concreto, explicitando la medida y el efecto que tiene la aplicación de cada uno de los criterios o factores que le permiten arribar a una sanción en específico. Este deber de fundamentación no implica la determinación ex ante o la existencia de un sistema de tarificación en materia ambiental, ya que solo comprende señalar en qué medida se ha aplicado un criterio, cuánto puntaje se le ha asignado, lo cual no limita la discrecionalidad en su determinación conforme a las bases metodológicas, y los motivos para esta, de manera que resulte posible reproducir el razonamiento que llevó a la SMA a determinar la sanción?7.

En particular, atendida la configuración de la fórmula establecida en las Bases Metodológicas:

[...] la asignación de puntaje y fundamentación de aquello resulta particularmente relevante respecto de factores que constituyen multiplicadores en la fórmula para la determinación de la sanción [...] este es el caso del valor de seriedad dentro del componente de afectación, pues su variación puede aumentar o disminuir de manera significativa la sanción aplicable . $^{8}$

Además, en opinión del tribunal el deber de determinar el puntaje sería también extensible a los factores de incremento o disminución, para efectos de reproducir el razonamiento que llevó a la SMA a la sanción específica, ejercer adecuadamente el derecho a defensa y posibilitar el adecuado control jurisdiccional que verifique si la sanción es proporcional o no.

En definitiva, el Segundo Tribunal Ambiental estimó configurado un vicio de legalidad en la sanción por falta de motivación, la cual se expresó en que la SMA no explicitó el puntaje asignado tanto al valor de seriedad, como a los factores de incremento y disminución de la sanción.

\footnotetext{
5 Fuenzalida Moure Compañía Limitada en contra de la Superintendencia del Medio Ambiente (2020): Segundo Tribunal Ambiental, c. $22^{\circ}$.

6 íbíd., c. $27^{\circ}$

7 Ibíd., c. $28^{\circ}$

8 Ibíd., c. $31^{\circ}$.
} 


\section{Sentencia de la Corte Suprema}

Contra la sentencia del Segundo Tribunal Ambiental tanto la SMA como FMC dedujeron recursos de casación en el fondo. En particular, la SMA reprochó el deber de motivación que impondría la sentencia del Segundo Tribunal Ambiental al exigir que la SMA exprese el valor numérico con el cual determinó la seriedad y circunstancias del artículo 40 de la LOSMA.

En lo que respecta a la motivación de la decisión sancionatoria exigida por el Segundo Tribunal Ambiental, la Corte Suprema luego de sintetizar los distintos reproches realizados a la fundamentación de las distintas circunstancias establecidas en el artículo 40 de la LOSMA, indicó que salvo el beneficio económico la SMA se limitó a señalar si las circunstancias serían consideradas o descartadas, sin precisar cómo influyen en el cálculo del componente de afectación, impidiendo el ejercicio del derecho a defensa y control jurisdiccional de proporcionalidad de la multa impuesta, afectando la fundamentación del acto administrativo, aspecto que es un elemento de su esencia y sujeto a su control.

Al respecto, resultan especialmente significativos los siguientes reproches:

no se señala, en concreto, cuáles son todas las razones que determinan el ejercicio de la potestad, incorporando incertidumbre respecto de los motivos que llevaron a determinar la cuantía de la multa reclamada, y cómo incidió en su determinación cada uno de los factores de incremento o disminución, en términos precisos y concretos" (...) "la fórmula utilizada en términos amplios por la Superintendencia del Medio Ambiente al dictar la resolución reclamada, vulnera gravemente los principios de publicidad y transparencia que debe regir la actuación de la Administración?.

[...] no es suficiente que se entreguen razones meramente formales, como ha ocurrido en la especie, en cuanto al componente de afectación, y, en particular, el valor de seriedad y factores de incremente y disminución, cuanto más si ellos han sido utilizado como principal factor en la determinación de la sanción específica finalmente impuesta, desde que los términos abstractos en que cada factor fue descritos (sic), no permite comprender la determinación de la autoridad fiscalizadora y reproducir el razonamiento que la llevó a imponer la sanción reclamada y su cuantía ${ }^{10}$.

Además, para la Corte Suprema la existencia de las Bases Metodológicas también implica un mayor estándar de fundamentación de sus actos y subsecuentemente, una revisión judicial más intensa.

\footnotetext{
9 Fuenzalida Moure y Compañía Ltda. con Superintendencia del Medio Ambiente (2021): CS, c. $16^{\circ}$. 10 Ibíd., c. $17^{\circ}$.
} 


\section{Algunas consideraciones sobre la regulación de la determinación de sanciones}

El entendimiento del debate suscitado entre FMC y la SMA requiere de la comprensión de algunos elementos regulatorios relativos a la determinación de sanciones ambientales.

La LOSMA estableció como organismo a cargo de la fiscalización y sanción de una serie de infracciones a ciertos instrumentos de gestión ambiental a la SMA ${ }^{11}$. Si bien la LOSMA estableció un amplio abanico de sanciones algunas de las cuales son severas ${ }^{12}$, en lo que respecta a la determinación de las sanciones su regulación es escueta.

La regulación puede resumirse en dos ideas esenciales, primero, la LOSMA establece sanciones disponibles según el rango de gravedad establecido en la propia norma (leve, grave y gravísima), así por ejemplo, una infracción gravísima puede ser objeto de revocación de la resolución de Resolución de Calificación Ambiental ("RCA"), clausura o multa de hasta 10.000 UTA, en cambio, una leve solo puede ser sancionada con una amonestación por escrito o una multa de hasta 1.000 UTA $^{13}$.

Además, la LOSMA identifica un listado de circunstancias o criterios que deben ser considerados por la SMA al determinar la sanción específica aplicable $^{14}$, pero no precisa cómo deben ser consideradas ${ }^{15}$. Además, el listado no es taxativo, pues incluye "Todo otro criterio que, a juicio fundado de la Superintendencia, sea relevante para la determinación de la sanción"16.

La literatura especializada coincide en que la LOSMA establece un amplio margen de discrecionalidad a la SMA para determinar sus sanciones ${ }^{17}$, el cual se encuentra guiado o condicionado ${ }^{18}$ por el marco legal ya descrito, que establece la materialización del principio de proporcionalidad ${ }^{19}$ en sede administrativa sancionatoria.

En otros términos, la determinación de una sanción ambiental por parte de la SMA corresponde a una potestad discrecional y como tal, se encuentra compuesta de elementos reglados y de apreciación discrecional ${ }^{20}$, que le permiten decidir entre una pluralidad de opciones o alternativas ${ }^{21}$.

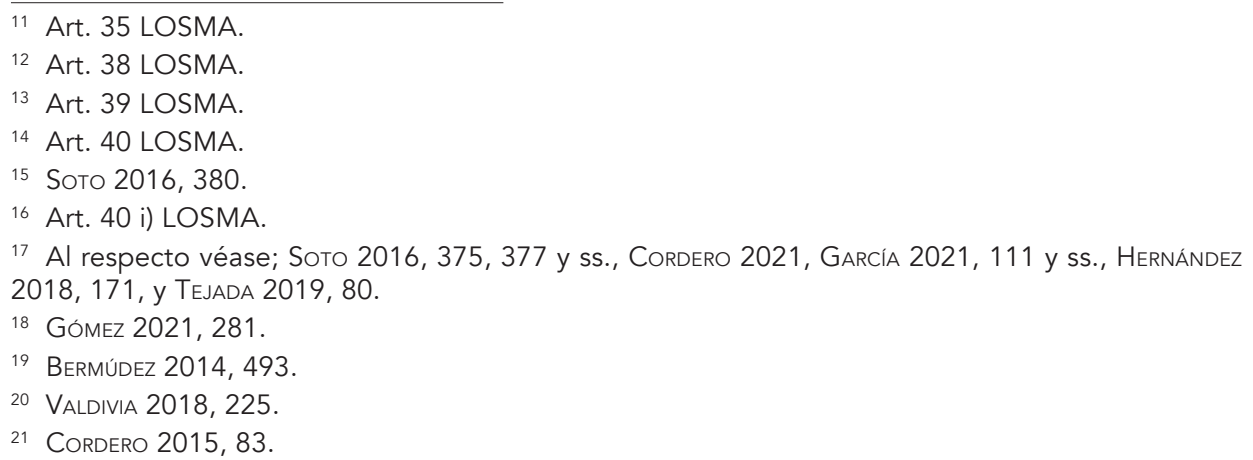


Lo anterior, lejos de implicar arbitrariedad o ser inconstitucional22, es precisamente lo que permite a la SMA ejercer de manera razonable su potestad sancionatoria ${ }^{23}$, pues le permite adecuarse a las circunstancias concretas de cada infracción.

Al respecto, cabe mencionar que la jurisprudencia ambiental se pronunció tempranamente clarificando la finalidad implícita en el carácter discrecional de la determinación de sanciones:

responde a que el posible infractor no pueda saber con antelación el beneficio de su inconducta, aumentando de esta forma el poder disuasivo del sistema de protección ambiental. La no entrega de la fórmula, desde esta perspectiva protege un interés ambiental ${ }^{24}$.

No obstante lo anterior, de manera pionera la SMA dictó en el año 2015 una primera versión de sus Bases Metodológicas ${ }^{25}$, la cual fue luego renovada el año $2018^{26}$ incorporando precedentes jurisprudenciales y actualizando ciertos criterios. Este documento establece tanto precisiones respecto de los criterios considerados por la SMA al momento de determinar una sanción, como un esquema metodológico que a través del uso de un polinomio ${ }^{27}$ permite arribar a los montos numéricos necesarios para la aplicación de las multas.

En síntesis, las Bases Metodológica establecen orientaciones relativas al cómo se implementan las circunstancias del artículo 40 de la LOSMA y como tal, estableció una suerte de confinamiento ${ }^{28}$ o autolimitación ${ }^{29}$ de la SMA respecto de sus propias potestades discrecionales al momento de determinar una sanción.

\section{Continuación y quiebre jurisprudencial}

Una singularidad del caso FMC es que dependiendo del prisma puede ser leído tanto como la consolidación de una línea de precedentes jurisprudenciales de la Corte Suprema sobre el estándar de motivación de los actos administrativos, como también un quiebre a la jurisprudencia ambiental en materia del tipo de fundamentación necesaria para la determinación de las sanciones por parte de la SMA.

\footnotetext{
22 Al respecto, cabe mencionar que este aspecto suscitó una reserva de constitucionalidad durante la tramitación de la LOSMA, véase: Hernández 2018, nota al pie Nº 1.

23 Gómez 2020, 198.

24 Empresa Nacional de Electricidad S. A. con Superintendencia del Medio Ambiente (2015):

Tercer Tribunal Ambiental, c. (101).

25 Resolución Exenta No 1002/2015 de la SMA.

26 Resolución Exenta N 85/2018 de la SMA.

27 Sото 2016, 375.

28 Sото $2016,393$.

29 GÓMEZ 2021, 313-314.
} 
Por un lado, tal como la doctrina ${ }^{30}$ ha señalado en la última década la jurisprudencia de la Corte Suprema ha saludablemente evolucionado robusteciendo progresivamente el escrutinio de la motivación exigible a los actos administrativos. Tal línea de precedentes que acentúan la rigurosidad del control de la motivación del acto, en vez de atenuarse con la entrada en vigor de los Tribunales Ambientales se ha profundizado ${ }^{31}$ y la decisión recaída en el caso FMC, puede ser comprendida como una muestra de ello.

En materia ambiental se evidencia una relación directa entre el grado de tecnicidad del control de la motivación y la rigurosidad de la motivación, en un extremo que no es necesariamente saludable, un ejemplo patente de ello es la decisión de la SMA en el caso de Pascua Lama con su extensión de más de dos mil páginas y diez anexos.

Por otro lado, paradójicamente la decisión en el caso de FMC establece un punto de inflexión respecto a precedentes ambientales relativos a la determinación de las sanciones ${ }^{32}$. Ello pues, tal como la propia SMA destacó de manera posterior al fallo ${ }^{33}$, la Corte Suprema en el caso de Empresa Nacional de Electricidad S.A. con SMA había asentado una diferencia entre las circunstancias del artículo 40 de la LOSMA de carácter cuantitativo y cualitativo, distinción que devenía en una forma distinta de motivar la sanción. Mientras las circunstancias cuantitativas daban pie para una expresión del monto, las cualitativas requerían "de un examen a la luz de los hechos específicos que fundan la sanción, por cuanto no es posible un cálculo exacto y ex ante de su incidencia" 34 .

Dicha distinción se difuminó en el caso de FMC, pues correspondería explicitar siempre en la resolución sancionatoria la cuantificación del puntaje asignado por la SMA a cada circunstancia, sin importar si el tenor de esta es cuantitativo o cualitativo.

Además, la decisión del caso FMC también pugna con lo sostenido por el Segundo Tribunal Ambiental a propósito del caso de Autódromo Codegua con SMA, donde se explicitó que

en la determinación de la sanción específica a aplicar en cada caso, la Superintendencia cumple el mandato legal cuando realiza una motivación adecuada de la necesidad de la sanción y de su cuantía, que haya tenido a la vista, y también cuando explica la influencia que cada uno

\footnotetext{
30 Al respecto véase; CORDERO 2021 y CORDERO 2017, 239.

31 TEJADA 2019, 78.

32 Siguiendo a Tejada 2019, 82-83, es posible considerar los siguientes fallos: Empresa Nacional de Electricidad S. A. con Superintendencia del Medio Ambiente (2015): Tercer Tribunal Ambiental; Eagon Lautaro S. A con Superintendencia del Medio Ambiente (2016): Tercer Tribunal Ambiental; y Municipalidad de Temuco con Superintendencia del Medio Ambiente (2016).

33 SMA 2021.

34 Empresa Nacional de Electricidad S. A. con Superintendencia del Medio Ambiente (2016): CS, c. 20.
} 
de los factores establecidos por la ley tienen sobre la sanción, lo que en caso alguno implica desarrollar una memoria de cálculo de la multa ${ }^{35}$.

\section{Germen de tarificación, riesgos y consecuencias}

La tarificación ambiental fue definida por la Corte Suprema precisamente en el caso de Empresa Nacional de Electricidad S.A. con SMA como: "el establecimiento de parámetros rígidos que permiten ex ante conocer el monto de las infracciones o, en otras palabras "cuánto cuesta" el infringir la normativa - se aleja de la finalidad preventiva que informa dicha institucionalidad" 36 .

No solo es tarificación la existencia de un listado cerrado de infracciones con sanciones determinadas ex ante (tarificación de sanciones en sentido estricto o fuerte). Sino que también, se produce tarificación si la rigidez del modelo de determinación de sanciones provee del conocimiento suficiente a los regulados para que sus predicciones sobre las consecuencias sancionatorias de sus infracciones resulten acertadas (tarificación de sanciones en sentido lato o débil). Alternativamente, si la aplicación del modelo deviene una suerte de fuente de conocimiento heurístico predictivo para los regulados.

En mi opinión, la decisión recaída en el caso FMC establece un origen o germen de esta última clase de tarificación. Si bien el conocimiento de los puntajes asignados a cada circunstancia o componente aplicado para la determinación de la sanción en el caso de FMC, por sí solo no produce un efecto predictivo, la aplicación de manera coherente y generalizada de dicho estándar por la SMA en todos sus procedimientos sí sería capaz de producir progresivamente tal efecto.

Para ello bastaría que los privados lleven un registro suficientemente exhaustivo de la aplicación de sanciones por parte de la SMA en las que explicite los puntajes. Una vez sistematizados suficientes casos sería posible prever de manera aproximada, por un lado, el rango de puntaje que cabe esperar para tal o cual circunstancia aplicable y reflejamente, las sanciones que se derivarían de dicha aplicación. Todo ello atendido que la función numérica que conjuga tales puntajes es pública y consta en las Bases Metodológicas.

Cabe enfatizar que el riesgo descrito es de carácter progresivo y el efecto predictivo es descrito como aproximado. Ello pues, tanto por la diversidad de instrumentos que tiene a su cargo la SMA para fiscalizar y sancionar, como la multiplicidad de maneras en que los mismos pueden ser trasgredidos y el indefinido ${ }^{37}$ conjunto de circunstancias que pueden llegar a concurrir en

\footnotetext{
35 Inversiones La Estancilla S. A. en contra de la Superintendencia del Medio Ambiente (2020): Segundo Tribunal Ambiental, c 64. En un sentido similar véase también la prevención del Ministro Ruiz en el caso: Eco Maule S. A. en contra de la Superintendencia del Medio Ambiente (2020): Segundo Tribunal Ambiental.

36 Empresa Nacional de Electricidad S. A. con Superintendencia del Medio Ambiente (2016): CS, c. 15².

37 La SMA siempre puede crear una o más circunstancias ad hoc para determinar la sanción en el caso concreto en virtud del artículo 40 i) de la LOSMA.
} 
un caso concreto incidiendo en la determinación de la sanción, continuará haciendo difícil encontrar casos completamente idénticos que sirvan de señalizadores perfectos.

Sin embargo, tal como la propia SMA reconoce hay ciertos instrumentos de gestión ambiental y tipos de infracciones ${ }^{38}$ derivados de los mismos, que es posible estandarizar o abordar masivamente. Atendidas las prácticas sancionatorias de la SMA y entre otras, las infracciones en materia de normas de emisión de ruido, residuos industriales líquidos y lumínica son candidatos obvios para que el germen de tarificación que advertimos se concretice primero.

A su vez, es importante advertir que el riesgo aquí esbozado no solo debilitaría la finalidad preventiva o disuasoria propia de las sanciones ambientales, pues al saber cuánto (aproximadamente) me costará tal o cual trasgresión, puedo infringir atendiendo a que la cuantía no es suficientemente disuasiva y/o que la posibilidad de detección es suficientemente baja. También podría empobrecer o a lo menos desviar las discusiones jurídico-ambientales en materia de sanciones.

Señalar el puntaje asignado a cada circunstancia puede implicar que las discusiones relativas a la determinación de sanciones tanto en sede administrativa como judicial se centren en si tal o cual puntaje numérico es elevado o menor, una suerte de regateo de multas entre privados y la SMA basado en los precedentes de esta última. Ello, en desmedro de focalizar el debate sancionatorio en la concurrencia o inconcurrencia de los supuestos fácticos o jurídicos necesarios para la aplicación de las circunstancias del artículo 40 de la LOSMA.

Ejemplos recientes de tal clase de situaciones que se han dado en un sector regulado distinto se observan en los casos de Chilquinta Energía S.A. con Superintendencia de Electricidad y Combustibles ("SEC") 39 y Transelec con SEC ${ }^{40}$. En este último, la propia Corte Suprema ordenó confeccionar un baremo de sanciones impuestas por la SEC para verificar si el castigo se encontraba dentro del rango promedio impuesto por la autoridad en precedentes similares.

Por otro lado, tal como la literatura especializada ha advertido el estándar exigido a propósito del caso FMC puede conllevar el efecto contrario al perseguido, "que el fiscalizador ambiental prefiera gestionar su discrecionalidad sancionatoria sin ningún tipo de autoconfinamiento metodológico" 41. Ello pues, la SMA podría derogar la resolución que aprueba las Bases Metodológicas y/o actualizarlas buscando que el control que pueda ejecutar la judicatura fundada en las mismas sea menor.

\footnotetext{
${ }_{38}$ Un ejemplo patente se encuentra en SMA 2020, 8, que establece tipos de infracciones asociadas a las normas de emisión del D.S. N 90/2000 y el D.S. N 46/2002.

39 Chilquinta Energía S. A. con Superintendencia de Electricidad y Combustible (2021): CS, c 14.

40 Transelec S. A. con Superintendencia de Electricidad y Combustible (2021): CS, c 5.

41 CoRdero 2021.
} 
Tal efecto inhibitorio también puede extenderse a otras agencias que desempeñan labores equivalentes a la SMA en otros sectores regulados y que incluso cuentan con marcos normativos similares ${ }^{42}$. A raíz del precedente de FMC, estas podrían preventivamente decidir no adoptar estrategias de autorregulación de manera pública y transparente a la ciudadanía en materia de determinación de sanciones ${ }^{43}$ como lo ha hecho la SMA.

\section{Conclusiones}

La LOSMA estableció tanto un amplio rango de sanciones como de discrecionalidad a la autoridad para determinarlas. Pese a ello, con el objeto de elevar el estándar de fundamentación de sus sanciones la SMA dictó las Bases Metodológicas, en ella estableció aplicar un polinomio para determinar las sanciones y, en consecuencia, confinó parcialmente sus potestades discrecionales.

La decisión de la Corte Suprema recaída en el caso FMC se funda en las Bases Metodológicas y a propósito de ellas, eleva el estándar de fundamentación requerido por parte de la SMA para la fundamentación de sus sanciones.

Lo anterior, si bien se enmarca en una serie de precedentes de la Corte Suprema, también establece una ruptura en lo que respecta a los precedentes jurisdiccionales propiamente ambientales, pues ahora resultaría necesario explicitar numéricamente los puntajes que se asigna a cada una de las circunstancias del artículo 40 que se invoque, ya sea a modo de factores de incremento, disminución o como integrantes del valor de seriedad, sin importar su carácter cualitativo o cuantitativo. En definitiva, se acotan aún más los márgenes de discrecionalidad de la SMA al momento de imponer una sanción.

Explicitar los puntajes en las sanciones de manera generalizada y coherente produce un germen de tarificación de las sanciones ambientales, esto es, que la aplicación del modelo de determinación de sanciones progresivamente se rigidice y los regulados sean capaces de predecir de una manera suficientemente aproximada las sanciones que les corresponderá a su actuar.

Lo anterior es perjudicial para el sistema de protección ambiental, no solo porque debilitaría el efecto disuasivo de las sanciones, sino que tanto en sede administrativa como judicial puede empobrecer las controversias en materia de determinación de sanciones.

Además, el precedente podría ocasionar el efecto contrario al pretendido tanto respecto de la propia SMA como de otras autoridades. Ello pues, la SMA podría reaccionar ampliando sus márgenes de discrecionalidad median-

\footnotetext{
42 Por ejemplo, la Ley $N^{\circ} 18.410$ que Crea la SEC en su artículo 16 también contiene un listado de circunstancias a considerar para la determinación de sanciones y la Ley $N^{\circ} 20.529$ Sistema Nacional de Aseguramiento de la calidad de la educación parvularia, básica y media y su fiscalización, dispone en sus artículos 73, 79 y 80, circunstancias análogas para la determinación de sanciones por parte de la Superintendencia de Educación.

${ }^{43}$ Un análisis sobre el estatus actual de autorregulación en el ordenamiento chileno a propósito de esta materia se puede encontrar en: Gómez 2021, 318 y ss.
} 
te la modificación o derogación de las Bases Metodológicas y/u otros organismos podrían optar por no dictar instrumentos análogos a las mismas.

Finalmente, para que la disuasión de una sanción sea óptima debe conjugarse apropiadamente la discrecionalidad de la autoridad y las garantías de los regulados. Algunos autores consideraron que la mera dictación de las Bases Metodológicas materializaba un régimen inusualmente garantista y de difícil justificación ${ }^{44}$, a mí parecer son decisiones como la recaída en FMC las que concretizan dicha advertencia.

\section{Bibliografía citada}

Bermúdez Soto, J. (2014). Fundamentos de Derecho Ambiental (2 ${ }^{\mathrm{a}}$ Ed.). Ediciones Universitarias de Valparaíso.

Cordero Vega, L. (2015). Lecciones de Derecho Administrativo (2 $2^{\mathrm{a}}$ Ed.). Thomson Reuters.

Cordero Vega, L. (2017). La motivación del acto administrativo en la jurisprudencia de la Corte Suprema. Revista de Estudios Judiciales (4), 231-250.

Cordero Vega, L. (30 abril 2021). La Motivación de la sanción administrativa ambiental. https://www.elmercurio.com/Legal/Noticias/Analisis-Juridico/2021/04/30/motivacion-de-sancion-administrativa-ambiental.aspx

Garcia Machmar, W. (2021). Introducción al procedimiento administrativo sancionador. Editorial Hammurabi.

Gómez GonzÁlez, R. (2020). Discrecionalidad y potestades sancionadoras de la Administración. Revista lus et Praxis, 26(2), 193-218.

Gómez GonzÁlez, R. (2021). Discrecionalidad y Potestad Administrativa Sancionadora Límites y Mecanismos de Control. Tirant lo Blanch.

HernÁndez Grimberg, M. P. (2018). Circunstancias Moderadoras de la Responsabilidad Ambiental en la aplicación de multas por la SMA. Anuario de doctrina y jurisprudencia Sentencias destacadas 2016 (14), 169-195.

Superintendencia del Medio Ambiente (5 de noviembre de 2015). Bases Metodológicas para la Determinación de Sanciones Ambientales. https://portal.sma.gob.cl/index. php/2015/11/05/sma-publica-bases-metodologicas-para-la-determinacion-de-sanciones/

Superintendencia del Medio Ambiente (2017). Bases Metodológicas para la Determinación de Sanciones Ambientales. https://portal.sma.gob.cl/index.php/download/bases-metodologicas-para-la-determinacion-de-sanciones-ambientales-2017/?wpdmdl=14238\&masterkey $=5$ be 1 ea5f3eac3

Superintendencia del Medio Ambiente (2021). Boletín de jurisprudencia Fiscalía Superintendencia del Medio Ambiente, 2(1), Primer cuatrimestre 2021. https://newsletter.sma. gob.cl/download/boletin-jurisprudencial-n4/?wpdmdl=13496\&masterkey=60bfa3dba4e34

Superintendencia del Medio Ambiente (2020). Guía para la presentación de programa de cumplimiento infracciones tipo a las normas de emisión de Riles. https://portal.sma.gob.cl/ index.php/download/guia-riles-2020/?wpdmdl=21738\&masterkey=5fa311 fabc0a1

Soto Delgado, P. (2016). Determinación de sanciones administrativas: disuasión óptima y confinamiento de la discrecionalidad del regulador ambiental. Anuario Derecho Público $\operatorname{UDP}(1), 2016,374-410$.

Tejada CAstillo, P. (2019). Discrecionalidad administrativa en la determinación de las sanciones ambientales. Revista de Derecho Ambiental (11), 56-87.

Valdivia Olivares, J. M. (2018). Manual de Derecho Administrativo. Tirant lo Blanch.

44 Sото 2016, 393. 


\section{Normativa citada}

Ley № 20.417 de 2010. Crea el Ministerio, el Servicio de Evaluación Ambiental y la Superintendencia del Medio Ambiente. 12 de enero 2010. D.O. N³9.570

Ley $N^{\circ} 18.410$ de 1985. Crea la Superintendencia de Electricidad y Combustibles. 26 abril 1985. D.O. N $\mathrm{N}^{\circ} 32.176$.

Ley N 20.529 de 2011. Sistema Nacional de Aseguramiento de la Calidad de la Educación Parvularia, Básica y Media y su Fiscalización. 11 agosto 2011. D.O. N 40.046.

\section{Jurisprudencia citada}

Empresa Nacional de Electricidad S.A. con Superintendencia del Medio Ambiente (2016): Corte Suprema, 13 de diciembre de 2016 (Rol No 17.736-2016). Tercera Sala [Recurso de casación].

Chilquinta Energía S. A. con Superintendencia de Electricidad y Combustible (2021): Corte Suprema. Tercera Sala Constitucional. Rol N 83664-2020, 12 febrero 2021.

Fuenzalida Moure y Compañía Ltda. con Superintendencia del Medio Ambiente (2021): Corte Suprema. Tercera Sala Constitucional. Rol № 79353-2020, 6 abril 2021.

Transelec S. A. con Superintendencia de Electricidad y Combustibles (2021) Corte Suprema. Tercera Sala Constitucional. Rol No38165-2021, 5 julio 2021.

Empresa Nacional de Electricidad S. A. con Superintendencia del Medio Ambiente (2015): Tercer Tribunal Ambiental, 27 de marzo de 2015 (Rol N R-6-2014).

Eagon Lautaro S. A. con Superintendencia del Medio Ambiente (2016): Tercer Tribunal Ambiental, 5 de febrero de 2016 (Rol N R-15-2015).

Municipalidad de Temuco con Superintendencia del Medio Ambiente (2016): Tercer Tribunal Ambiental, 23 de marzo de 2016 (Rol N R-22-2015).

Eco Maule S. A. en contra de la Superintendencia del Medio Ambiente (2020): Segundo Tribunal Ambiental, 29 de abril de 2020 (Rol N R-174-2018).

Fuenzalida Moure Compañía Limitada en contra de la Superintendencia del Medio Ambiente (2020): Segundo Tribunal Ambiental, 1 de junio de 2020 (Rol Nº R-196-2018).

Inversiones La Estancilla S. A. en contra de la Superintendencia del Medio Ambiente (2020): Segundo Tribunal Ambiental, 4 de septiembre de 2020 (Rol N R-195-2018). 
